\title{
EL VARIADOR DE VELOCIDAD COMO MÉTODO DE ARRANQUE IDEAL PARA MOTORES ELÉCTRICOS DE INDUCCIÓN
}

(The variable speed drive VSD as ideal method for starting electric induction motors)

\section{Julio Alberto Carrillo Romero, Jairo Alberto Rojas Boyacá}

Universidad Pedagógica y Tecnológica de Colombia. Grupo de Investigación DSP

juliocarrilloromero@yahoo.es jairoarojasb@hotmail.com

(Recibido19 de Noviembre de 2012 y aceptado 13 de Marzo de 2013)

\section{Resumen:}

Mediante la programación de parámetros, la puesta en marcha del variador de velocidad de referencia CFW-11, y la realización de arranques con motores eléctricos de inducción, de diferentes características, se analiza el comportamiento de las variables: voltaje, corriente, torque y velocidad en el motor, para corroborar que el método de arranque con variador es el ideal. Se obtienen gráficas del sistema, por medio de la implementación de la herramienta Trend del software SuperDriveG2, las cuales se analizan para determinar el comportamiento de la aplicación.

Palabras clave: variador de velocidad, motor de inducción, convertidor de frecuencia.

\section{INTRODUCCIÓN}

En el arranque de un motor eléctrico intervienen variables físicas que están asociadas a la dinámica del sistema, como el voltaje y la corriente, las cuales deben ser controladas para evitar oscilaciones del sistema. De ahí la importancia de contar con información sobre la naturaleza de las magnitudes que intervienen en el proceso. Con la información obtenida se diseñan estrategias de control que ayudan al cumplimiento de un objetivo específico.

Una adecuada programación en un variador de velocidad, permite reducir el pico máximo de corriente de arranque y las caídas de tensión en la red, los golpes mecánicos y el desgaste en los acoplamientos, lo cual se ve reflejado en las magnitudes de salida, que, por medio del software, se pueden medir, hacer seguimiento online y representar gráficamente.

En el mercado industrial hay programas que admiten hacer aplicaciones con variadores de velocidad, tal como se indica en la tesis de grado Diseño y construcción de un módulo con variador de frecuencia para el control de velocidad de motores asincrónicos jaula de ardilla trifásicos para el laboratorio de control industrial, de Cárate y Villacís (2011). Para fines educativos y de este artículo, se usa un variador de frecuencia de marca WEG y referencia CFW-11, el cual es programado y puesto en marcha con tres motores de diferentes características. Se utiliza el software SuperDrive G2 9.40, que es un programa para parametrización, control, seguimiento online y representación gráfica de las magnitudes que intervienen en el proceso de arranque de un motor de inducción, como son: voltaje, corriente, torque y velocidad. Con el análisis de estas gráficas se puede establecer si la programación efectuada en el variador está o no acorde con los requisitos del sistema.

\section{Abstract:}

By programming parameters, implementation of VSD reference CFW-11, and conducting starts with electric induction motors, with different characteristics, we analyze the behavior of the variables: voltage, current, torque and engine velocity to verify that the starting VSD method is the ideal. System graphs are obtained by implementing the tool "Trend" from SuperDriveG2 software, which are analyzed to determine the behavior of the application.

Keywords: variable speed drive VSD, induction motor, variable frequency drive.

\section{SELECCIÓN DE DATOS Y PROGRAMACIÓN}

\subsection{Herramientas}

Módulo con variador industrial, motores eléctricos de inducción, computador portátil, software SuperDrive G2.

\subsection{Métodos de arranque para motores eléctricos}

El arranque de motores eléctricos es uno de los principales problemas que presenta perturbaciones y caídas de tensión en la red de distribución, debido a las altas corrientes de arranque y a que la rápida aceleración del sistema motor-carga requiere un elevado par de arranque, lo que repercute negativamente en la vida útil del motor. Por tal razón es necesario controlar estas magnitudes, pues no se desea crear perturbaciones en la red, y si se producen grandes aceleraciones en el arranque, se corre el riesgo de dañar los engranajes o acoples del sistema.

Existen distintos métodos para el arranque de motores eléctricos de inducción. A continuación se hace una comparación de los más importantes:

\subsubsection{Arrancador directo:}

El motor es conectado directamente a la tensión nominal de la red, a través de un dispositivo de protección y control llamado arrancador o contactor. En este tipo de arranque, se producen unos valores muy elevados en el par e intensidad, los cuales pueden ser el doble y hasta siete veces el valor nominal respectivamente.

\subsubsection{Arrancador estrella-triángulo:}

Esta conexión consiste en arrancar el motor conectado en estrella, y, una vez que alcanza la velocidad de trabajo, esto es, 607 segundos después, se conecta en triángulo. 
Con esta aplicación, el par e intensidad desciende a menos de un tercio respecto al arranque en conexión directa. Aunque en la transición de estrella a triángulo se genera el mismo efecto que en el arranque directo, la variación es que el efecto dura menos tiempo.

\subsubsection{Arrancador suave:}

El motor es conectado a un dispositivo electrónico que permite limitar el tiempo de arranque, la rampa de corriente y tensión de salida, hasta 300 o $400 \%$ para la corriente nominal, y entre 30 y $70 \%$ para la tensión de salida. Tiene muchas ventajas con respecto a los dos anteriores, pues genera menor perturbación en la red y el arranque es controlado por rampa de tensión, de corriente o una combinación tensión/corriente.

\subsubsection{Variador de velocidad:}

El motor se conecta por medio de un dispositivo electrónico de potencia, que posibilita efectuar arranque y parada de manera controlada, variando la velocidad y el par de fuerza del motor, mediante la alteración de la frecuencia aplicada al motor, convirtiendo las magnitudes fijas de frecuencia y tensión de red, en magnitudes variables. Para lograr esto, rectifica la tensión alterna de red, y por medio de transistores o IGBT's, trabajando en modulación de ancho de pulso, genera una corriente trifásica de frecuencia y tensión variable, que permite controlar la aceleración y el par de arranque del motor.

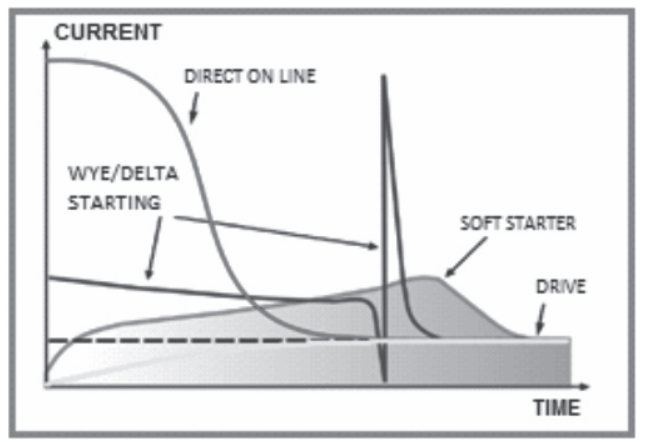

Figura 1. Comportamiento de la corriente en el motor a diferentes métodos de arranque. Fuente: (WEG, 2011

En la Figura 1 se identifica claramente la ventaja que representa el arranque de motores con variador, comparado con los otros métodos, pues los demás presentan picos de corriente de arranque, que generan sobresfuerzos en los conductores y exigen un mayor par de arranque en el motor, que se traducen en la red, en perturbaciones y caídas de tensión; en el proceso, en golpes y desgastes mecánicos; y en el motor, en calentamiento y desgaste prematuro de los acoplamientos.

\subsection{Selección de datos}

Es necesario hacer una correcta selección de los datos que se ingresan en la programación del variador, estos hacen referencia al motor, al proceso y al variador. En seguida se detalla cuáles corresponden a cada uno.

\subsubsection{Datos del variador:}

En la Figura 2 se observa la placa de características del convertidor de frecuencia. De la placa de características del convertidor, se puede destacar la siguiente información:
- Voltaje nominal 220 - 240 VAC 3 .

- Corriente nominal 7 Amp.

- Frecuencia 50/60 Hz.

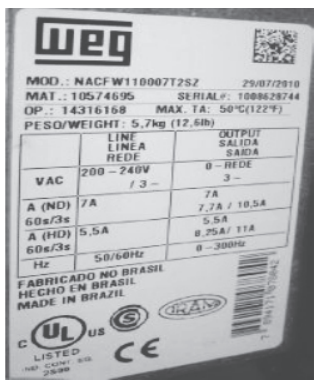

Figura 2. Placa de características variador WEG CFW-11. Fuente: los autores.

\subsubsection{Datos de los motores:}

Las características de los motores se encuentran en la placa de identificación, como se anota en el artículo "Selección y aplicación de motores eléctricos" (WEG, 2011). Hay que prestar especial atención al tipo de aislamiento, corriente nominal, factor de servicio, tensión nominal, eficiencia, velocidad y frecuencia, pues estos datos son necesarios para una adecuada programación del variador, sin llegar a afectar la vida útil del motor.

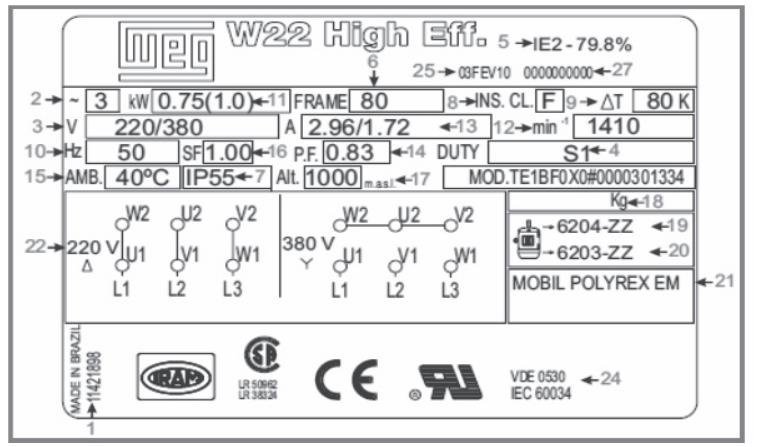

Figura 3. Modelo de una placa de características para un motor de inducción trifásico. Fuente: www.weg.com.

En la Figura 3 se aprecia el modelo de placa de un motor con todas sus características, las cuales son descritas en el “Catálogo técnico de motores W22" de WEG (2011), y en el artículo "Motores Eléctricos" (PEMEX, 2005).

Los datos de placa de características para los tres motores utilizados en la práctica, están relacionados en la Tabla 1:

Tabla 1. Datos nominales de los motores usados para las pruebas

\begin{tabular}{lccl}
\hline Magnitud & Motor 1 & Motor 2 & Motor 3 \\
\hline Potencia HP & 0.5 & 0.5 & 2.3 \\
Voltaje 3 $~$ & 220 & 220 & 220 \\
Corriente & 2.07 & 2.32 & 6.4 \\
Frecuencia Hz & 60 & 60 & 60 \\
SF & 1.15 & 1.15 & 1 \\
P.F. & 0.69 & 0.71 & 0.82 \\
INS. CL. & F & F & F \\
Velocidad Rpm & 1680 & 1130 & 3420 \\
\hline \multicolumn{2}{r}{ Fuente: los autores. } & &
\end{tabular}

\subsubsection{Datos del proceso:}

Información referente al proceso, necesaria para hacer una adecuada programación del variador, por tratarse de un
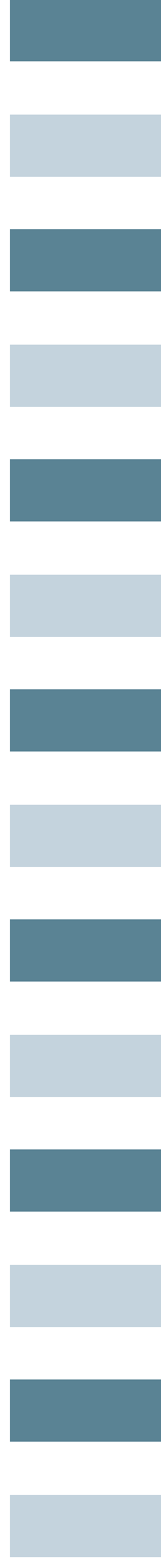

\section{.}


proyecto de uso educativo y por no tenerse la infraestructura necesaria. Se asume que el proceso requiere un arranque y parada de forma controlada. Dependiendo del tipo de aplicación que se demande, deberá tenerse información adicional, que, por lo general, está determinada o limitada por el proceso, como es: tiempo y rampa de arranque, tipo de desaceleración, tipo de carga, tipo de torque; además debe definirse si la aplicación arranca con carga o en vacío, a fin de efectuar una correcta programación de parámetros en el variador. Los datos necesarios para programar el variador, son:

- Tensión de red 220 VAC.

- Tiempo de aceleración 20 segundos.

- Tiempo de desaceleración 20 segundos.

- Tipo de control V/F $60 \mathrm{~Hz}$.

- Tipo de rampa aceleración.

- Arranque en vacío/sin carga.

Es conveniente tener en cuenta que la potencia, la corriente y la tensión de operación del motor, así como del variador, se encuentren en el mismo rango, además se recomienda que la potencia del variador sea superior a la del motor en un $10 \%$.

\subsection{Programación de parámetros en el variador}

La programación de parámetros en el variador se puede hacer por medio de la IHM incorporada en el módulo o por un computador portátil vía cable USB. Para efectos de mayor aprendizaje, se hará por medio del PC. El software permite realizar la programación de parámetros y hacer comparación de estos, respecto de los valores máximos y mínimos que son programados, así como de los valores que vienen predeterminados de fábrica.

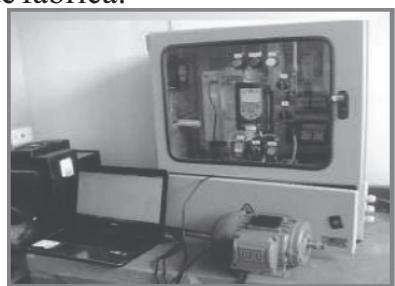

Figura 4. Módulo con variador de velocidad industrial CFW-11. Fuente: los autores

En la Figura 4 se contempla el módulo con variador de velocidad, un motor y el PC que contiene el software SuperDrive $G 2$, utilizado para la práctica.

La interfaz es bastante amigable y fácil de trabajar, en su pantalla de inicio permite seleccionar el modelo del variador con el cual se trabaja, se configura el tipo de comunicación y selecciona el rango de operación de la aplicación en voltaje/corriente.

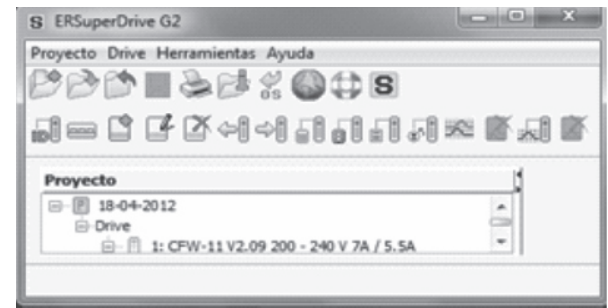

Figura 5. Pantalla general software SuperDrive G2. Fuente: los autores.

La Figura 5 muestra la pantalla general del software, que contiene una serie de iconos en la parte superior, los cuales admiten desarrollar diferentes actividades.
2.4.1 Funciones principales software SuperDrive G2.

Las más importantes son:

New parameters file: permite crear un nuevo grupo de parámetros y guardarlo en un archivo.

Read parameters from drive: lee todos los parámetros del drive, para guardarlos en la computadora. Permite visualizar o editar parámetros.

Write parameters to drive: envía todos los parámetros de la computadora al drive. Algunos parámetros pueden ser modificados solamente con el drive deshabilitado (motor parado).

Monitor parameters: programa y monitorea online todas las variables y algunos parámetros de la aplicación.

Monitor parameters: programa y monitorea online todas las variables y algunos parámetros de la aplicación.

\section{Monitor status: identifica el estado del drive}

Monitor speed reference: visualiza los parámetros asociados con la frecuencia del drive.

Trend: grafica variables de salida, permite seleccionar y configurar sus valores máximos, mínimos y genera tendencias.

Trace: configura variables de salida, para que cuando ocurra una falla, estas sean graficadas.

Con los datos de placa del motor del variador, la información necesaria sobre las condiciones de arranque y conociendo cómo funciona el software de programación, se procede a programar el variador, (Carrillo \& Rojas, 2012). Para el caso y aplicación específica, se modifican los parámetros relacionados en la Tabla 2 .

Tabla.2. Parámetros más relevantes modificados en el variador de velocidad. Fuente: los autores.

\begin{tabular}{|c|c|c|c|}
\hline Păám. & Función & Valor & Unidades \\
\hline P 100 & Tiempo aceleración & 20 & Segundos \\
\hline P 135 & $\begin{array}{l}\text { Corriente máxima de } \\
\text { salida }\end{array}$ & 3 & $\begin{array}{l}1,5 \times \text { I } \\
\text { nominal }\end{array}$ \\
\hline P 156 & Corriente sobrecarga & 2,4 & $1.15 \times$ In \\
\hline P 169 & $\begin{array}{l}\text { Máxima corriente } \\
\text { Torque }+\end{array}$ & 100 & $\%$ \\
\hline P 202 & Tipo de control & 0 & $\mathrm{~V} / \mathrm{f} 60 \mathrm{~Hz}$ \\
\hline P 295 & $\begin{array}{l}\text { Corriente nominal de } \\
\text { ND/HD }\end{array}$ & 3 & $7 \mathrm{~A} / 5.5 \mathrm{~A}$ \\
\hline P 296 & $\begin{array}{l}\text { Tensión nominal de la } \\
\text { red }\end{array}$ & 0 & $220-240 \mathrm{~V}$ \\
\hline P 398 & $\begin{array}{l}\text { Factor de servicio del } \\
\text { motor }\end{array}$ & 1.15 & \\
\hline P 399 & Rendimiento del motor & 90 & $\%$ \\
\hline P 400 & $\begin{array}{l}\text { Tensión nominal del } \\
\text { motor }\end{array}$ & 220 & Voltios \\
\hline P 401 & $\begin{array}{l}\text { Corriente nominal del } \\
\text { motor }\end{array}$ & 2.1 & Amperios \\
\hline P 402 & $\begin{array}{l}\text { Rotación nominal del } \\
\text { motor }\end{array}$ & 1680 & Rpm \\
\hline P 403 & $\begin{array}{l}\text { Frecuencia nominal del } \\
\text { motor }\end{array}$ & 60 & $\mathrm{~Hz}$ \\
\hline P 404 & $\begin{array}{l}\text { Potencia nominal del } \\
\text { motor }\end{array}$ & 0.5 & HP \\
\hline P 406 & Ventilación del motor & 0 & Autoventilado \\
\hline P 407 & $\begin{array}{l}\text { Factor de potencia } \\
\text { nominal del motor }\end{array}$ & 0,69 & \\
\hline
\end{tabular}


Los parámetros modificados en la Tabla 2, se refieren a las condiciones necesarias por el proceso, a las características nominales del motor, a las características del tipo de arranque y del variador. Se modifica la programación para dos motores más, cuyos datos están relacionados en la Tabla 1.

\section{ANÁLISIS DE RESULTADOS}

Por medio de la función Trend, resultan las gráficas 6, 7 y 8, donde se evidencia el comportamiento de las magnitudes físicas más relevantes en el proceso de arranque de los motores, las cuales son: voltaje, corriente, velocidad y torque de salida o aplicado al motor.

\subsection{Curvas características del sistema}

Como se observa en las gráficas 6,7 y 8 , la corriente de arranque no sufre ningún pico, al contrario, va aumentando progresivamente hasta llegar a estabilizarse en su valor normal de operación. Esto demuestra que el arranque de motores con variador de velocidad es el método ideal, pues se logra una armonía en los tres elementos involucrados, el motor, el proceso y la red de distribución, desarrollando la actividad sin afectar ningún componente de los mismos.

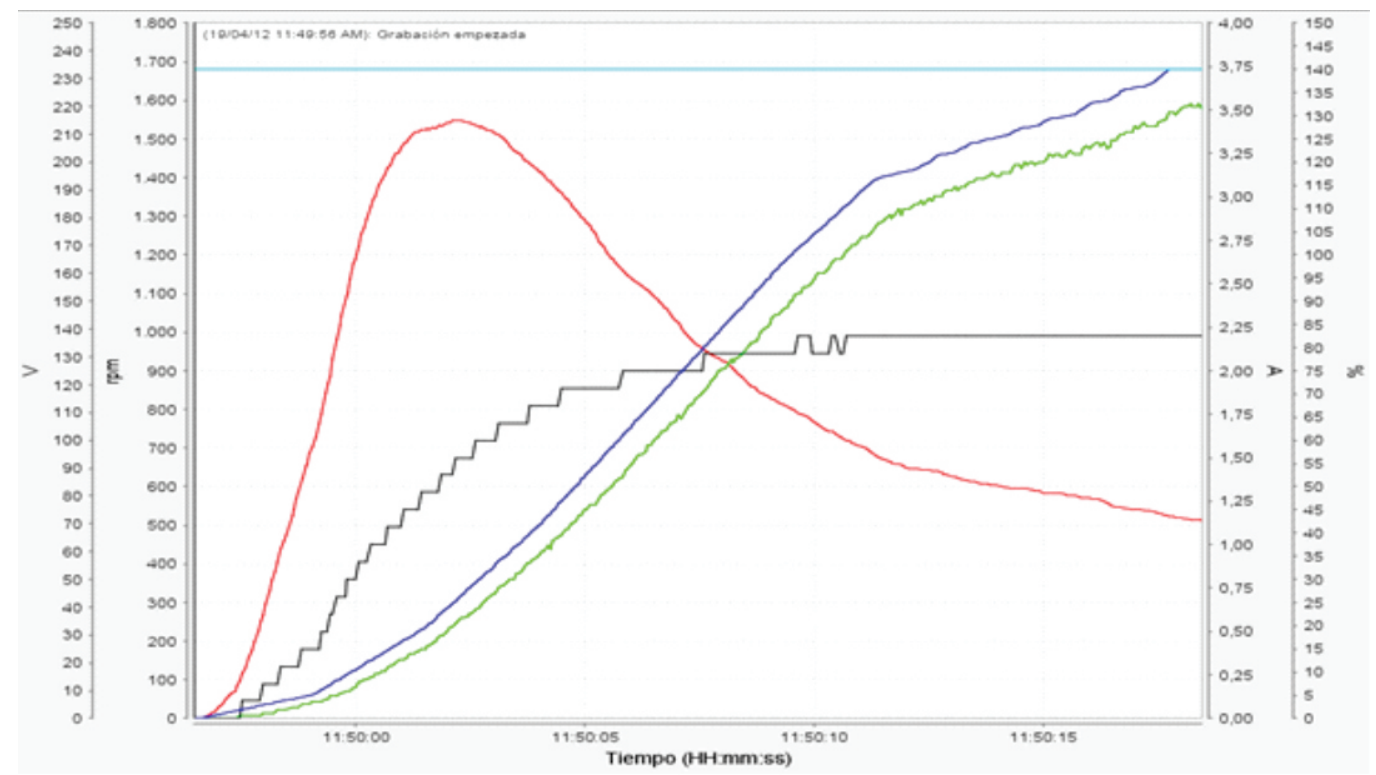

Figura 6. Curva de arranque en 20 segundos para el motor de 0.5 hp/1680 rpm. Fuente: los autores.

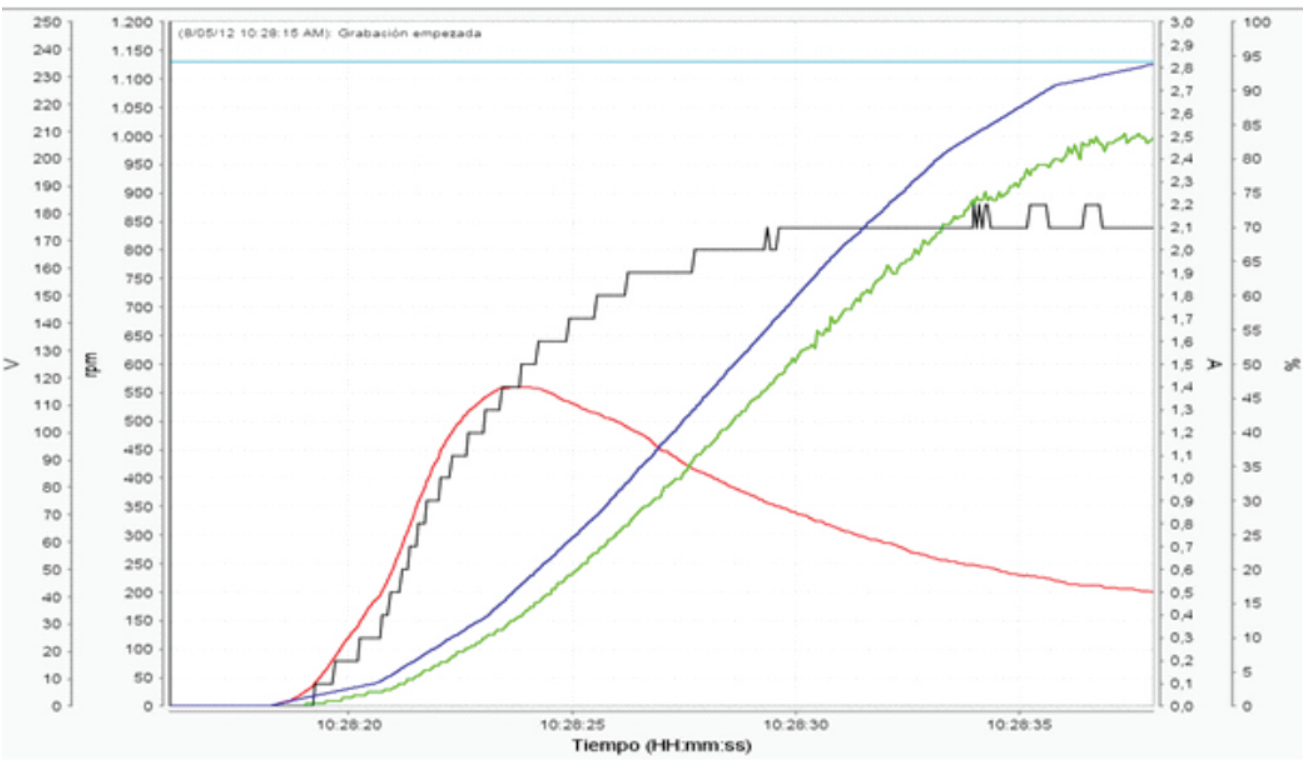

Figura 7. Curva de arranque en 20 segundos para el motor de $0.5 \mathrm{hp} / 1130 \mathrm{rpm}$. Fuente: los autores. 
En la Figura 6 se observa que el torque, aunque presenta un pico entre los 4 y 6 segundos posteriores al arranque, no es tan alto como en los otros métodos, en los que puede alcanzar hasta el $200 \%$ de su valor normal. A medida que la velocidad del motor aumenta, el torque disminuye hasta su valor de operación, lo que demuestra que el torque y la eficiencia son inversamente proporcionales a la velocidad del motor, pues la Figura 6 representa la curva del motor con menor eficiencia y se aprecia que el pico del torque es mayor, de igual manera que su valor de operación normal.

En la Figura 7 se nota que la velocidad y el voltaje en el motor aumentan linealmente, desde el cero hasta su valor nominal de operación.

La Figura 8 muestra que el motor alcanza su corriente de operación aproximadamente a la mitad del voltaje y velocidad del mismo, segundos después de que el torque empieza a descender de su punto más alto.

\subsection{Comparación datos de salida de los tres motores}

En la Tabla 3 se recopilan los datos más relevantes de la información obtenida por medio de las gráficas de arranque.

Tabla.3. Cuadro comparativo arranque de tres motores a 20 segundos

\begin{tabular}{|c|c|c|c|c|c|}
\hline & $\begin{array}{r}\text { Tiempo } \\
\text { segundos }\end{array}$ & Voltaje & Corriente & Rpm & $\begin{array}{c}\text { Torque } \\
\%\end{array}$ \\
\hline & 5 & 26 & 1,3 & 244 & 127 \\
\hline $\begin{array}{l}\text { Motor } 1 \\
0.5\end{array}$ & 10 & 103 & 2 & 841 & 89,6 \\
\hline$\frac{0,5 \mathrm{HP}}{1680}$ & 15 & 180 & 2,2 & 1406 & 55,1 \\
\hline \multirow{2}{*}{$\begin{array}{l}1680 \\
\text { Rpm }\end{array}$} & 20 & 216 & 2,2 & 1674 & 43,8 \\
\hline & máx & 223 & 2,2 & 1680 & 129 \\
\hline & 3 & 26 & 1,3 & 163 & 45,6 \\
\hline $\begin{array}{l}\text { Motor } 2 \\
0.55 P\end{array}$ & 10 & 98 & 2 & 566 & 33,2 \\
\hline 1130 & 15 & 173 & 2,1 & 959 & 21,5 \\
\hline \multirow[t]{3}{*}{$\underset{\mathrm{Rpm}}{\mathbf{n}}$} & 20 & 208 & 2,1 & 1128 & 16,6 \\
\hline & máx & 216 & 2,2 & 1130 & 46,7 \\
\hline & & 24 & 2,2 & 501 & 13,1 \\
\hline Motor 3 & 10 & 91 & 2,3 & 1696 & 6,6 \\
\hline & 15 & 165 & 2,4 & 2887 & 6,3 \\
\hline \multirow{2}{*}{$\begin{array}{l}3420 \\
\mathbf{R p m}\end{array}$} & 20 & 200 & 2,5 & 3412 & 6,1 \\
\hline & máx & 205 & 2,5 & 3420 & 18,8 \\
\hline
\end{tabular}

La Tabla 3 muestra los valores medidos en el arranque de los motores. El valor de la corriente nunca excede el valor nominal de operación de los motores; el voltaje y velocidad aumentan linealmente y llegan a su valor nominal de operación, después de los 20 segundos programados en el arranque; el motor 3 ,

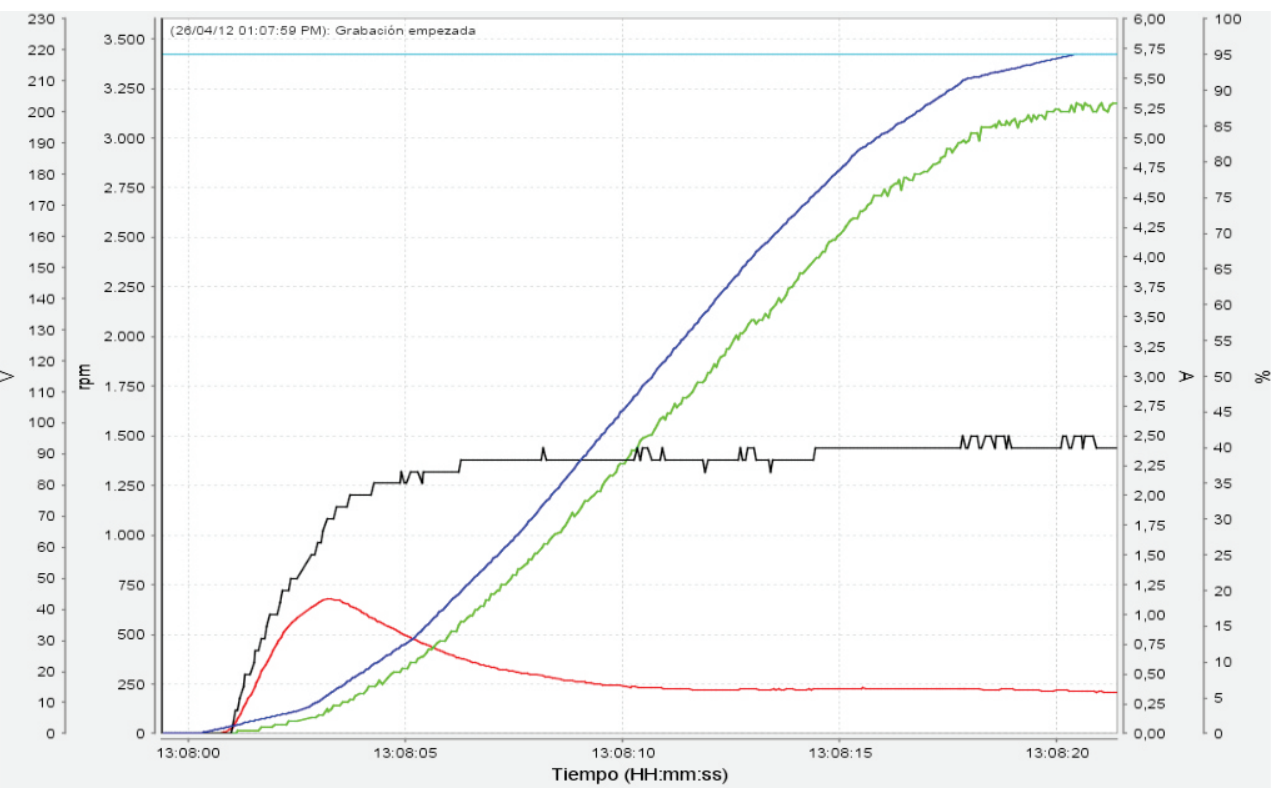

Figura 8. Curva de arranque en 20 segundos para el motor de $2.3 \mathrm{hp} / 3420 \mathrm{rpm}$. Fuente: los autores

cuya eficiencia es mayor, presenta el menor valor de torque aplicado al motor, tanto en el arranque como en operación continua.

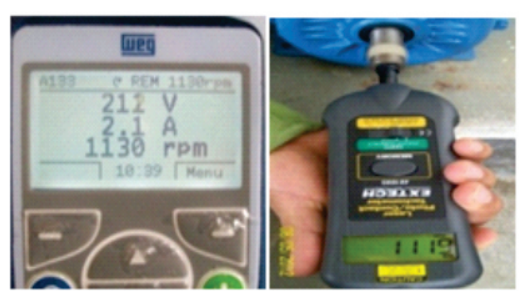

Figura 9. Medida de velocidad en el variador y corroborada con tacómetro digital para el motor de $0.5 \mathrm{hp} / 1130 \mathrm{rpm}$. Fuente: Los Autores.

Se mide la velocidad con tacómetro digital, la cual se observa en la Figura 9, donde se corrobora la velocidad real de salida del motor con los datos de la IHM del variador. El deslizamiento máximo registrado fue de 11 RPM, aunque se corrige a solo 2 RPM a máxima velocidad.

\section{CONCLUSIONES}

Se concluye que el variador de velocidad es el método más efectivo en el arranque de los motores eléctricos,

pues no presenta sobrepicos de corriente, y, de esta manera, no se afecta ni se crean perturbaciones en red. Por otra parte, los arranques se pueden hacer controladamente, evitando golpes y desgastes en los componentes mecánicos.

Se comprueba que para la programación del variador, no solo son necesarios los datos de placa del motor, pues también intervienen variables del proceso que se va a controlar, así como parámetros propios del variador, los que hay que tener muy en cuenta, si se quiere efectuar una adecuada programación. 
Por medio del software SuperDrive G2, se obtienen gráficas de las curvas características del arranque de diferentes motores, las cuales permiten realizar un análisis detallado de las condiciones y magnitudes que intervienen en el arranque de un motor eléctrico de inducción.

\section{REFERENCIAS}

Cárate, A.O. \& Villacís, A.R. (2011). Diseño y construcción de un módulo con variador de frecuencia para el control de velocidad de motores asincrónicos jaula de ardilla trifásicos para el laboratorio de control industrial. Tesis de grado. Riobamba: Escuela Superior Politécnica de Chimborazo, Facultad de Mecánica, p. 3-20.

Carrillo, J.A. \& Rojas, J.A. (2012). Guía básica para la programación y operación de un variador de velocidad industrial $C F W-11$ para su uso en ambientes educativos. Trabajo de grado. Sogamoso: Universidad Pedagógica y Tecnológica de Colombia, Escuela de Posgrados, p. 30-58.
Gaguancela, X.O. \& Sáez, G. (2011). Dotación y aplicación de un modulo con variador de velocidad para simulación de control de arranque y velocidad del motor de una grúa para el laboratorio de control industrial. Tesis de grado.

Riobamba: Escuela Superior Politécnica de Chimborazo, Facultad de Mecánica, p. 3-19.

PEMEX. (2005). Motores Eléctricos. Documento NRF-095PEMEX-2004. Recuperado de www.pemex.comhttp:// .

WEG. (2008). Guía de usuario CFW-11. Documento 10000063156. Recuperado de www.weg.nethttp://.

WEG. (2011). Automatización arrancadores suaves. Recuperado de www.weg.nethttp://.

WEG. (2011). Catálogo técnico de motores. Documento 50024297. Recuperado de www.weg.nethttp://.

WEG. (2011). Selección y aplicación de motores eléctricos. Recuperado de www.weg.nethttp://.

Yax, D.O. (2009). Automatización de cuartos fríos para ahorro energético. Tesis de grado. Guatemala: Universidad de San Carlos de Guatemala, Facultad de Ingeniería, p. 79-111. 